\title{
Cost of hospitalisation for hip fracture-findings from the Irish hip fracture database
}

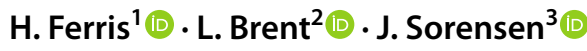

Received: 1 November 2021 / Accepted: 29 December 2021 / Published online: 11 January 2022

(c) International Osteoporosis Foundation and National Osteoporosis Foundation 2022

\begin{abstract}
Summary The authors utilised the Irish Hip Fracture Database (IHFD) to quantify the impact of hip fracture on the health service in terms of incidence, bed days and financial costs. The absolute number of hip fracture cases recorded by the IHFD has increased, as has the associated costs of hospitalisation.

Introduction Hip fracture places a considerable clinical and financial burden on the healthcare system, with acute hospitalisation accounting for a substantial proportion of the costs incurred. This paper aimed to quantify the cost of hospitalisation for hip fracture in Ireland in terms of bed days and direct hospital costs.

Methods The authors analysed 23,494 cases in the Irish Hip Fracture Database (IHFD) from 2014 to 2020. Case numbers and length of stay were analysed annually. Hospital costs for hip fracture were described using the 2020 Activity-Based Funding Price List, which outlines the fees paid to public hospitals for inpatient activity.

Results For the time period 2014-2020, the total cost of hospitalisation for hip fracture was approximately €296 million, equating to approximately $€ 11,700$ per episode of care. The annual cost of hospitalisation increased from approximately $€ 34$ million in 2014 to $€ 44$ million in 2020. In 2020, the mean length of stay for hip fracture was 17 days accounting for $>62,600$ acute hospital bed days.

Conclusion The absolute number of hip fracture cases recorded by the IHFD has increased, as has the cost of hospitalisation. Given the current capacity issues and economic constraints, there is a growing need to prioritise time spent in the most expensive acute hospital setting to the immediate perioperative period and maximise the use of community services and early supportive discharge for the rehabilitation phase.
\end{abstract}

Keywords Bed days $\cdot$ Costs $\cdot$ Economic burden $\cdot$ Financial costs $\cdot$ Hip fracture $\cdot$ Length of stay

\section{Introduction}

Hip fracture places a considerable burden on society in terms of morbidity, mortality and healthcare utilisation. Ireland has one of the highest age standardised incidence rates of hip fracture in the world at a rate of 406/100,000 for females [1]. In real terms, this equates to $>3,600$ cases per annum, with this figure set to increase in parallel with the

H. Ferris

ferrish@tcd.ie

1 Department of Public Health, Health Service ExecutiveSouth, Cork, Ireland

2 National Office of Clinical Audit, Dublin, Ireland

3 Healthcare Outcomes Research Centre, RCSI University of Medicine and Health Sciences, Dublin, Ireland aging population [2]. The direct costs associated with the acute management of hip fracture are substantial as surgical fixation and hospitalisation are key components of the care pathway for the majority of hip fracture patients.

The absolute number of all fragility fracture admissions increased by $30 \%$ between 2000 and 2014, with hip fracture accounting for a third of all admissions and almost half of all bed days [3]. Previous research has shown that healthcare costs for hip fracture patients are three times higher than age matched counterparts [4]. This has considerable implications for the health service as the total direct and indirect cost of falls and fractures for people aged $\geq 65$ years is estimated at $€ 404$ million per annum, accounting for $4.2 \%$ of all public health expenditure in Ireland [5]. As life expectancy continues to increase, so will the incidence of hip fracture with the associated need for hospitalisation, rehabilitation and community care. Robust data on the impact of hip fracture 
on healthcare utilisation is essential to facilitate efficient health service planning and the delivery of cost-effective patient care.

\section{Aim}

To identify the annual cost of inpatient care for hip fracture in Ireland 2014-2020 in terms of bed days and direct hospital costs.

\section{Methods}

\section{Data source}

The Irish Hip Fracture Database (IHFD) is a clinically led, web-based system where data are collected though the Hospital In-Patient Enquiry (HIPE) system, in association with the Healthcare Pricing Office (HPO). The audit is clinically supported by the Irish Institute of Trauma and Orthopaedic Surgery (IITOS) and the Irish Gerontological Society (IGS). Operational management is provided by the National Office for Clinical Audit (NOCA). The IHFD is a timely and accurate database with $99 \%$ coverage in recent years.

The Irish Hip Fracture Standards (IHFS) are the standards against which the Irish healthcare services are benchmarked. The IHFS are in alignment with the Best Practice Tariff (BPT) for hip fractures, which focuses on 8 core parameters: 6 clinical and 2 for data quality and clinical governance. IHFS 7 for early mobilisation is the latest standard to be introduced to the IHFD and will be included in the BPT from 2022.

\section{Data collection \& data entry}

The IHFD collects data on hip fracture patients through a specific IHFD portal on the HIPE system. The HIPE system is the principal source of demographic, clinical and administrative information on all discharges and deaths from publicly funded acute hospitals in Ireland [6]. All sixteen trauma units in the Republic of Ireland voluntarily submit data on patients that are discharged following hip fracture. Data from patients' medical records are entered retrospectively in each hospital by audit coordinators, with support from the local IHFD clinical lead and NOCA. A specific data entry form is used to record details of an extensive number of variables ranging from the patients' pre-operative functional status to the type of surgery and clinical outcomes. Data are collected for each hip fracture episode from admission to hospital discharge. Eligibility criteria are as follows:

Inclusion criteria: (i) Diagnosis on HIPE of either hip fracture due to injury (ICD-10-AM diagnosis codes S72.00 to S72.2) or with a specified type of fracture (e.g. intracapsular displaced, intracapsular undisplaced, intertrochanteric, subtrochanteric or open) other than periprosthetic.

(ii) Aged 60 years or over.

\section{Data quality \& data validation}

Data from HIPE are merged with additional IHFD data and an anonymised extract is sent to NOCA from the HPO. The data is assessed within NOCA using internationally agreed dimensions of data quality [7]. Data Validation Reports (DVR) are distributed to each hospital along with quarterly reports. The NOCA data analytics team verify the data with the contributing hospitals and HPO. Data quality is monitored through monthly meetings with the local audit coordinators and regular two-way feedback. Once data is validated, an annual report is produced and sent to the IHFD governance committee for approval. The IHFD data dictionary is available in the most recent IHFD annual report [2].

\section{Patient population}

Within the IHFD, patient demographics and case mix have remained largely unchanged over the time period 2014-2020. However, the most notable change in the patient population over the study period is an increase in the degree of frailty and comorbidity of patients, i.e. ASA Grade III (severe systemic disease) admissions increased from $39 \%$ in 2013 to $55 \%$ in 2020. A full description of the trends in hip fracture care in the Republic of Ireland has been published elsewhere [8]. The majority of Irish hip fracture patients are females aged 80 years or older who sustain a hip fracture from low energy trauma i.e. fall less than $2 \mathrm{~m}$. Home is the most common source of admission (85\%). The most common type of surgical repair is cemented hemi-arthroplasty (36\%) followed by internal fixation with a short IM nail (18\%) [2]. Spinal anaesthesia continues to be the predominant type of anaesthesia, either on its own (55\%) or in combination with a nerve block (21\%) [2]. Patient demographics, clinical characteristics and care pathway are outlined in Table 1.

\section{Activity-Based Funding}

Since 2016, Public hospitals in Ireland are funded using Activity Based Funding (ABF), as opposed to block funding. With this model of funding, hospitals are paid a fixed amount for inpatient and day case activity based on Diagnosis Related Groupings (DRG). Cases that are clinically similar and that are expected to consume a similar amount 
Table 1 Patient characteristics and care pathway for Irish hip fracture patients 2020 [2]

\begin{tabular}{|c|c|}
\hline Parameter & Percentage \\
\hline \multicolumn{2}{|l|}{ Gender } \\
\hline Female & $67 \%$ \\
\hline Male & $33 \%$ \\
\hline Mean age & 81 \\
\hline Over 80 years & $43 \%$ \\
\hline \multicolumn{2}{|l|}{ Admission source } \\
\hline Home & $85 \%$ \\
\hline Nursing home & $11 \%$ \\
\hline Acute hospital transfer & $4 \%$ \\
\hline \multicolumn{2}{|l|}{ Cognitive status on admission } \\
\hline Normal cognition & $69 \%$ \\
\hline Cognitive impairment $(\mathrm{AMT}<7)$ & $31 \%$ \\
\hline \multicolumn{2}{|l|}{ Pre-fracture mobility } \\
\hline Low functional ability & $53 \%$ \\
\hline High functional ability (NMS $>6$ ) & $47 \%$ \\
\hline \multicolumn{2}{|l|}{ Previous fragility fracture } \\
\hline Yes & $35 \%$ \\
\hline No & $65 \%$ \\
\hline \multicolumn{2}{|l|}{ Bone health } \\
\hline Bone health medication pre-admission & $13 \%$ \\
\hline Bone health medication started during admission & $59 \%$ \\
\hline Awaiting dual energy $x$-ray absorptiometry scan & $5 \%$ \\
\hline Assessed - no bone protection needed & $6 \%$ \\
\hline Not assessed & $9 \%$ \\
\hline Awaiting out-patient assessment & $8 \%$ \\
\hline \multicolumn{2}{|l|}{ Type of trauma } \\
\hline Low energy & $95 \%$ \\
\hline High energy & $2 \%$ \\
\hline Unknown & $3 \%$ \\
\hline \multicolumn{2}{|l|}{ ASA grade } \\
\hline I or II & $37 \%$ \\
\hline III & $55 \%$ \\
\hline IV or $\mathrm{V}$ & $8 \%$ \\
\hline \multicolumn{2}{|l|}{ Fracture type } \\
\hline Intracapsular displaced & $40 \%$ \\
\hline Intracapsular undisplaced & $8 \%$ \\
\hline Intertrochanteric & $37 \%$ \\
\hline Subtrochanteric & $6 \%$ \\
\hline Other & $9 \%$ \\
\hline \multicolumn{2}{|l|}{ Type of anesthetic } \\
\hline Spinal only & $55 \%$ \\
\hline Spinal and nerve block & $21 \%$ \\
\hline GA only & $13 \%$ \\
\hline GA and nerve block & $8 \%$ \\
\hline GA and spinal & $3 \%$ \\
\hline \multicolumn{2}{|l|}{ Type of surgery } \\
\hline Arthroplasty hemi cemented & $36 \%$ \\
\hline Internal fixation - IM nail short & $18 \%$ \\
\hline Internal fixation - IM nail long & $14 \%$ \\
\hline
\end{tabular}

Table 1 (continued)

\begin{tabular}{ll}
\hline Parameter & Percentage \\
\hline Internal fixation - dynamic hip screw & $12 \%$ \\
Arthroplasty hemi uncemented & $12 \%$ \\
Arthroplasty THR cemented & $3 \%$ \\
Arthroplasty THR uncemented & $2 \%$ \\
Internal fixation screws & $1 \%$ \\
Other & $2 \%$ \\
Irish Hip Fracture Standards (IHFS) & \\
IHFS 1 (Admitted to orthopedic ward <4 h) & $33 \%$ \\
IHFS 2 (Surgery within 48 h) & $75 \%$ \\
IHFS 3 (Pressure ulcer) & $3 \%$ \\
IHFS 4 (Review by Geriatrician) & $82 \%$ \\
IHFS 5 (Bone health assessment) & $91 \%$ \\
IHFS 6 (Specialist falls assessment) & $85 \%$ \\
IHFS 7 (Early mobilisation by Physiotherapist) & $78 \%$ \\
Length of stay & \\
Mean & 17 days \\
Median & 11 days \\
Discharged home directly & $28 \%$ \\
In hospital mortality & $5 \%$ \\
\hline
\end{tabular}

of resources are grouped into DRG [9]. Each DRG is represented by a number indicating a medical or surgical procedure, plus a code indicating the level of complexity. The price assigned to each DRG is applicable to the entire episode of care from admission to discharge. Outpatient activity and Emergency Department activity is not included. Ireland is currently using version 8.0 of the Australian Refined DRG system.

\section{Analysis}

We analysed 23,494 cases in the IHFD from 2014 to 2020. Data were exported from Microsoft Excel into Stata ${ }^{\circledR}$ (version 17) for analysis. Length of stay was measured as the number of calendar days from admission to discharge from hospital. The annual number of bed days in the acute hospital was calculated by multiplying the mean length of stay by the absolute number of hip fracture cases in the given year. Direct hospital costs were calculated using the 2020 ABF Price List from the HPO. Eight DRG codes were used for hip fracture patients: I03 A/B, I08 A/B, I78 A/B and W02 A/B. The 2020 list price for each specific procedure was multiplied by the number of episodes recorded by the IHFD. A small proportion of cases were coded as 'other'. In these cases, the case weighted average price of the eight procedure codes used for hip fracture patients was applied. Logistic regression analysis was conducted to investigate the association between ASA Grade and length of stay. 


\section{Results}

\section{Hip fracture incidence}

Since 2014 , there has been a $36 \%$ increase in the absolute number of hip fractures with data recorded by the IHFD (Fig. 1). However, this is in the context of data completeness in the IHFD increasing from $84 \%$ in 2014 to $95 \%$ in 2017 and $>99 \%$ thereafter. A slight reduction in incidence was seen in 2020 during the COVID 19 pandemic.

Although the absolute number of hip fracture cases has increased in recent years, incidence rates have declined both in Ireland and globally. The age standardised rates for the susceptible population in Ireland have declined over the last decade from 538 to 423 per 100,000 for females and from 285 to 225 per 100,000 for males [10]. The reasons for this are not fully understood.

\section{Length of stay-international trends}

There is considerable variation from country to country in terms of length of stay in an acute hospital following hip fracture. This is most likely due to different patient demographics, case mix, care pathways and healthcare systems. For instance, the mean (median) length of stay in Spain is 11 (9) days, UK 16 (12) days, Australia 9 (8) days and a mean of 8 days for both Sweden and Denmark (Fig. 2) [11]. The USA (Kaiser Permanente) has the shortest reported mean length of stay of 5 days. In contrast, Ireland has a longer mean length of stay of 20 (13) days. However, Germany reports the longest median length of stay at 16 days. As not all of the hip fracture registries report both the mean and median values, mean length of stay only is depicted in Fig. 2.

\section{Length of stay-national trends}

There was little fluctuation in length of stay during the time period 2014-2019 (Fig. 3). However, a notable decline was

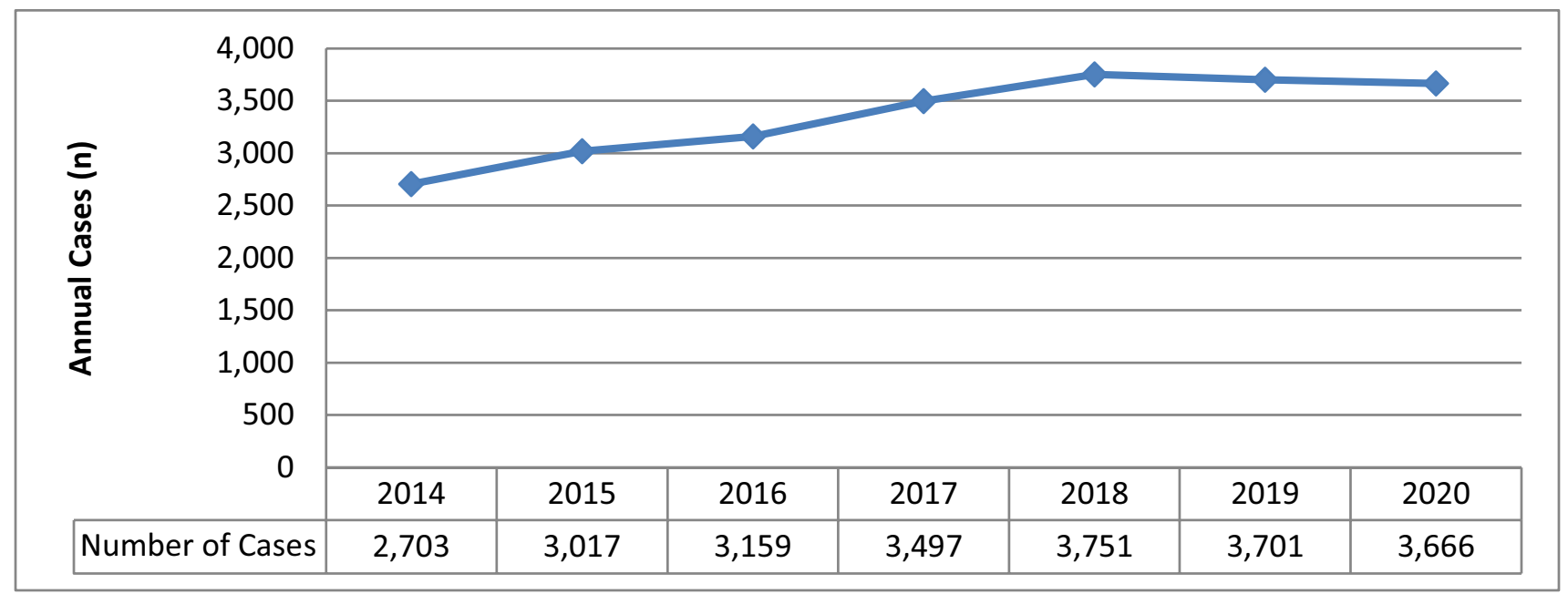

Fig. 1 Hip fracture cases in Ireland 2014-2020

Fig. 2 International overview of mean length of stay (days) 2019 [9]

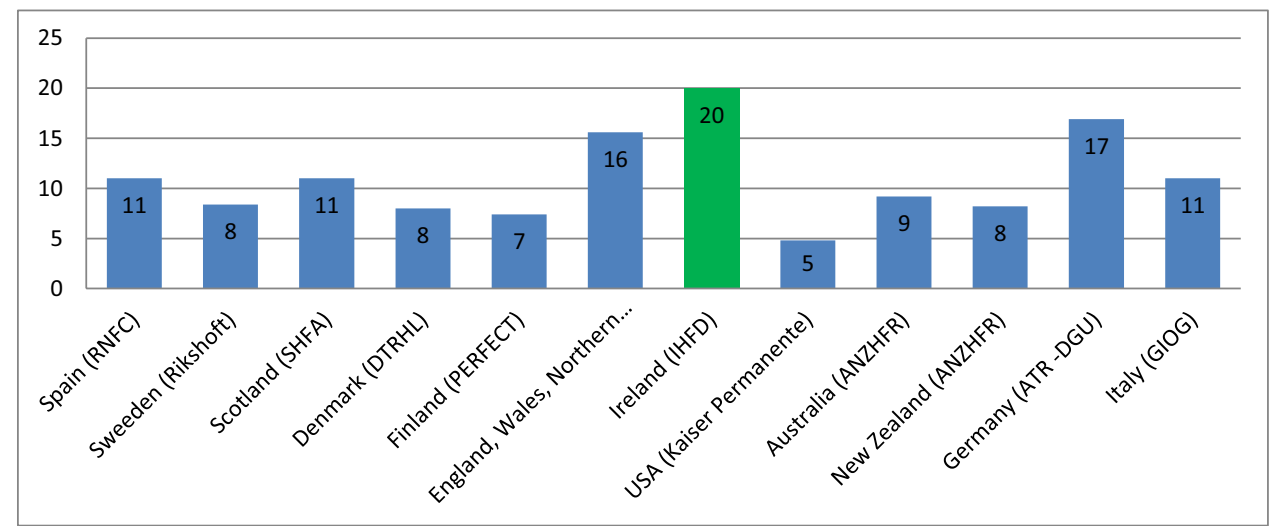


Fig. 3 National trends in length of stay 2014-2020

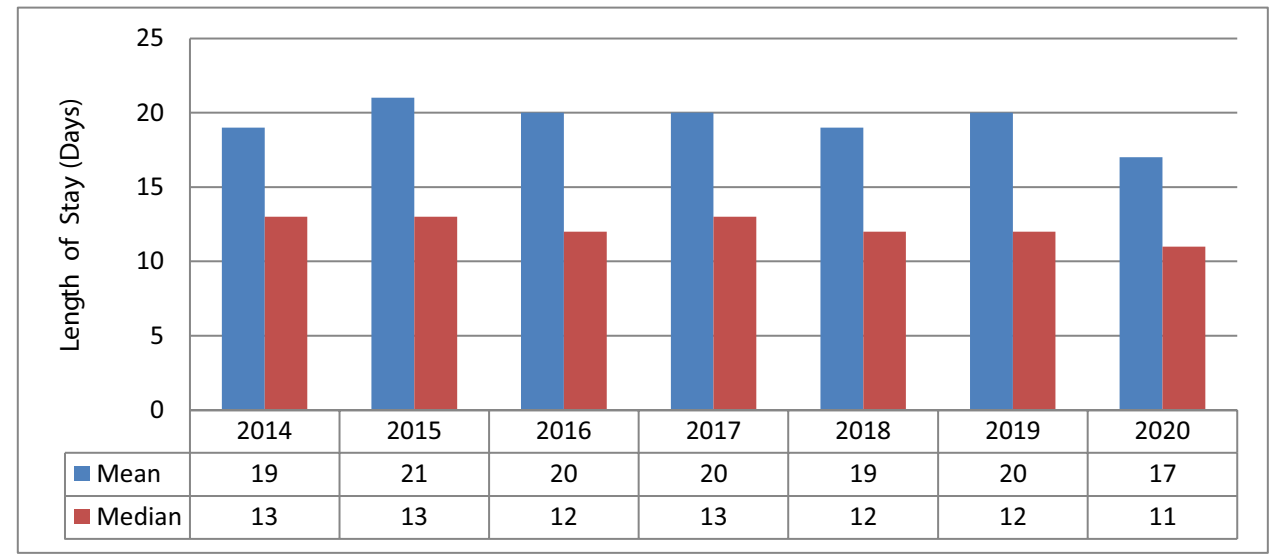

seen in 2020 with a reduction in the mean (median) length of stay from 20 (12) days in 2019 to 17 (11) days in 2020, which equated to a saving of $\sim 10,000$ bed days. This substantial reduction is attributed to new internal patient pathways and greater communication between the acute hospitals and the community sector during the COVID 19 pandemic. More specifically, there was an increase in the number of internal rehabilitation beds, greater involvement with community intervention teams and early supported discharge teams, as well as an increase in transfers to off-site facilities such as private hospitals and stand-alone orthopaedic hospitals [12]. This collaborative effort resulted in a shorter length of stay and a greater proportion of patients discharged home directly.

Within the IHFD, patients with higher ASA Grades had longer lengths of stay. Logistic regression analysis showed that patients with severe systemic disease (ASA Grade 3) were 3 times more likely to stay longer than the mean length of stay in hospital (OR 3.01, 95\% CI 2.39-3.78, $p<0.001$ ) (Supplementary Table 1). Similarly, those with ASA Grade 4 disease were 4.5 times more likely to stay longer than the mean length of stay (OR 4.57, 95\% CI 3.56-5.86, $p<0.001$ ).
This is important as length of stay impacts on bed days and overall cost of hospitalisation.

\section{Bed days}

Hip fracture places a considerable burden on the acute hospital sector. In 2020 alone, the mean length of stay for hip fracture was 17 days accounting for $>62,600$ bed days (Fig. 4).

\section{Direct costs of inpatient care}

The direct hospital costs of hip fracture care are tabulated using the 2020 ABF Price List from the HPO, which outlines the fee paid to public hospitals for inpatient activity (Table 2). Hip replacement of minor complexity was the most common procedure code used for hip fracture patients. For the time period 2014-2020, hospitalisation for hip fracture cost the Irish health service approximately $€ 296$ million, which equates to a cost of approximately $€ 11,700$ per episode of care. The cost of hospitalisation increased over the study period from approximately $€ 34$ million in 2014 to
Fig. 4 Acute hospital bed days for hip fracture 2014-2020

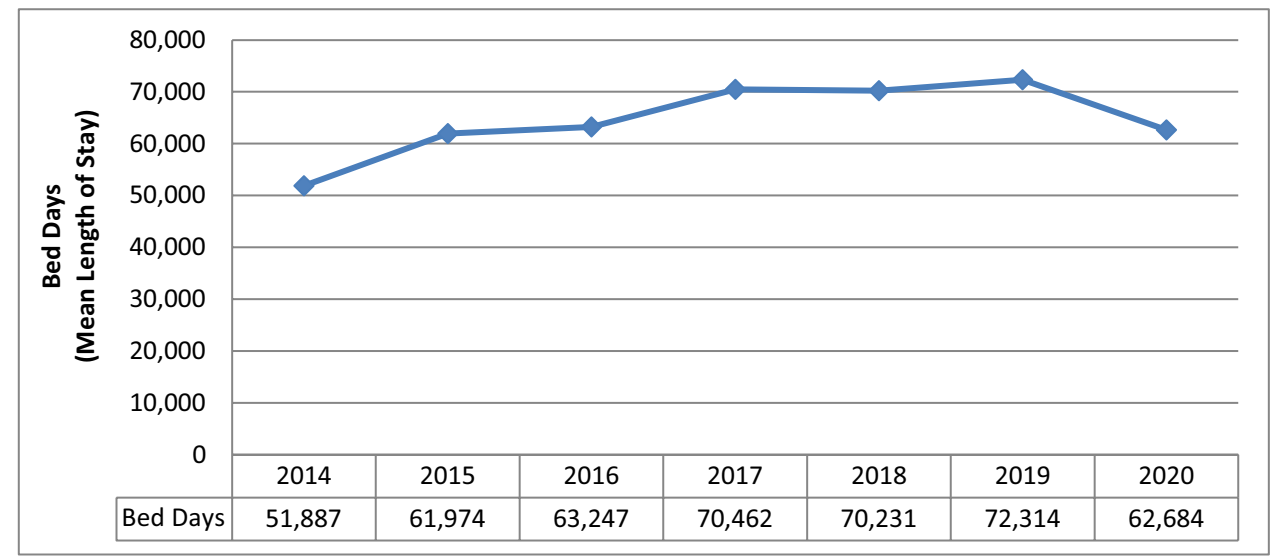




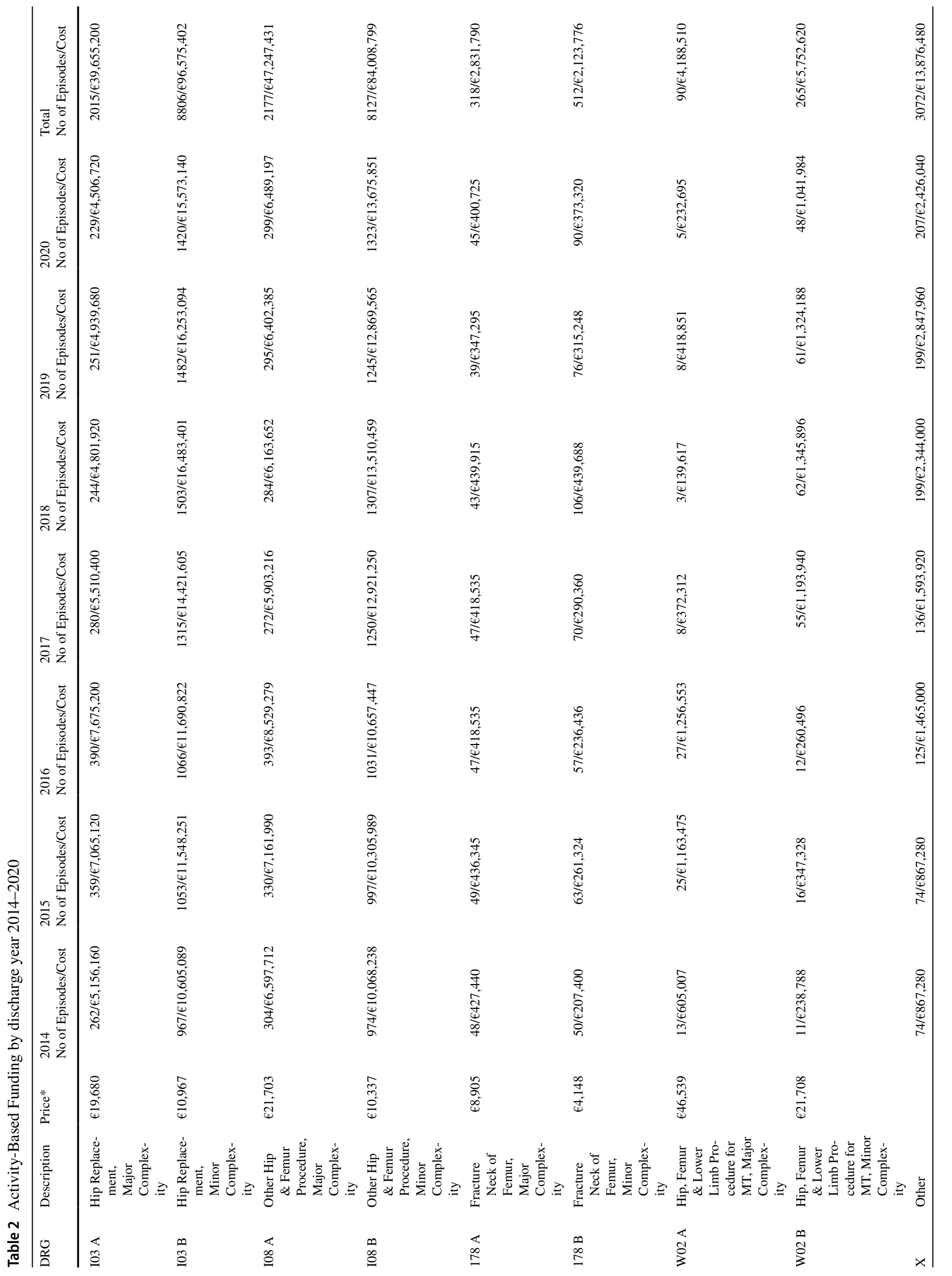


$€ 44$ million in 2020 . This $28 \%$ increase in costs over a 6 year period is substantial.

\section{Discussion}

Hip fracture places a considerable clinical and financial burden on the healthcare system, with acute hospitalisation accounting for a substantial proportion of the costs incurred. Since 2014, there has been a $36 \%$ increase in the absolute number of hip fractures recorded by the IHFD. The cost of hospitalisation for hip fracture 2014-2020 was approximately $€ 296$ million, equating to approximately $€ 11,700$ per episode of care. This finding is in keeping with previous research showing an average cost of $€ 12,687$ per admission in Ireland [6]. Similarly, in Italy, the cost of acute hospitalisation for hip fracture is $€ 10,079$ [13]. In the UK, hospitalisation costs associated with admission for primary hip fracture are approximately $£ 8,663$ [14]. In a systematic review and meta-analysis of over 100 studies looking at the cost of fragility fractures globally, Williamson et al. showed a pooled estimated cost of $\$ 10,075$ for the index hospital admission [15].

Although, the costs of hospitalisation are similar from country to country, the staggering costs involved in hip fracture care provides a stark incentive to look at more sustainable ways of delivering healthcare at the lowest level of complexity once the patient is medically fit for discharge. Recently, Ferris et al. highlighted how not only is Discharging Directly Home (DDH) integral to the quality of life of patients; it is also a key driver of the socioeconomic cost of hip fracture. In terms of the modifiable factors, patients who are mobilised early post operatively are $24 \%$ more likely to be DDH (OR 1.24, $p<0.01,95 \%$ CI 1.06-1.45) [16]. Conversely, patients who experience surgical delay are less likely to be DDH (OR $0.70, p<0.01,95 \%$ CI $0.56-0.88$ ).

Timely surgery can impact length of stay and reduce the cost of admission. Castelli et al. analysed data from $>60,000$ hip fracture patients across 152 hospitals in the UK and showed that costs are lower for those who received surgery on the same day of admission and discharged to their own home [17]. Currently, only 75\% of Irish hip fracture patients are operated on within $48 \mathrm{~h}$ of admission [2]. This is in contrast to countries such as Germany and Sweden where 72\% and $66 \%$ of patients respectively are operated on within $24 \mathrm{~h}$ of admission [18]. An Orthogeriatric model of care has also been shown to reduce time to surgery, improve the overall quality of care and enhance patient outcomes [19].

In Ireland, the IHFS are the quality standards against which services are benchmarked. The IHFS encompass process measures that improve patient outcomes, i.e. prompt admission to an orthopaedic ward, timely surgery and early post-operative mobilisation [20]. Hospitals that 
adhere to the IHFS are rewarded with a Best Practice Tariff, which is a financial incentive for providing high quality patient care. A payment of $€ 1000$ is paid to the hospital for each case that meets the IHFS. $70 \%$ of this payment goes to the trauma service and 30\% to hospital management. Since its introduction in 2018, the number of hospitals qualifying for the BPT has increased year on year. Financial payments of $€ 278,000$ were made in 2018 , which increased to $€ 548,000$ in 2019 and to $€ 710,000$ in 2020 [2]. The IHFS and BFT serve to improve patient outcomes by optimising the care pathway, reducing the length of stay in the higher acuity hospital setting and enabling patients to return to independent living following hip fracture. Further analysis of the impact of the BPT on costs and bed days will be conducted when more years of data are available.

Length of stay is often used as a surrogate marker of the efficiency of a service. In recent years length of stay has remained relatively stable at a mean (median) of 20 (17) days despite an increase in the degree of frailty of the patient cohort. An improvement was seen in 2020 during the COVID pandemic when there was greater emphasis on early supported discharge and more effective communication between the various teams involved in the care of hip fracture patients. Given that hip fracture accounts for $>62,600$ bed days per year at considerable cost to the health service, there is a strong economic incentive to identify cost effective approaches to the management of hip fracture [21]. It is important to note that in addition to differences in the patient population and clinical practice, some of the variation in length of stay internationally may be due to differences in the place of residence pre-fracture. Ireland et al. showed that hip fracture patients admitted from residential care have widely different lengths of stay compared to those admitted from the community, with delays in transfer to a rehabilitation facility being the major obstacle [22]. Salonga-Reyes et al. also highlighted how the lack of available supported care beds, residential care beds and difficulties in obtaining funding for home care packages compounds the issue of delayed discharge from an acute hospital [23].

Finally, but perhaps most importantly, hip fracture is preventable. Given that the majority of hip fractures occur as a result of a low impact fall in the home, it is imperative that we support older adults to stay safe in their own environment. During the COVID 19 pandemic, many people were spending more time in their homes, which prompted NOCA to devise some practical tips for older adults on how to stay safe at home (Supplementary Fig. 1). Promoting bone health through education on the importance of diet and exercise, as well as early identification and treatment of osteoporosis are key to improving the health outcomes of those at risk of sustaining a fragility fracture.

\section{Limitations}

This paper utilises a large national database of older adults to describe the impact of hip fracture on the acute hospital system and associated financial costs. Although the IHFD was established in 2012, data completeness in the earlier years was sub-optimal, therefore the authors analysed the database from 2014 to 2020.

Data pertaining to diagnoses and procedures is coded directly from patients' hospital records by trained HIPE coders. The accuracy of the HIPE system is dependent on the accuracy of the discharge summary, which is completed by a member of the medical team at time of discharge. Although the HIPE system is open to human error, it is the only record of acute hospital admissions in Ireland.

Direct hospital costs for hip fracture were calculated using the ABF Price List from the HPO, which assigns a fixed value to the episode of care from admission to discharge based on DRG. ED attendance and outpatient visits are not included in the DRG system, therefore the costs outlined in this paper do not capture the full patient journey. In addition, costs are not calculated separately for specific individual comorbidities, as this information is not captured by the IHFD. ASA grade is used as an indication of the burden of systemic disease. In the absence of a Unique Health Identifier, it is not possible to take other costs such as ambulance transportation or rehabilitation into consideration.

\section{Conclusion}

This paper utilised the IHFD to quantify the impact of hip fracture on the Irish health service in terms of bed days and direct hospital costs. The absolute number of registered hip fracture cases has increased and places a considerable burden on the acute hospital system at a substantial financial cost. There is a strong economic incentive to identify cost effective approaches to the management of hip fracture at the lowest level of complexity such as early supported discharge and discharge directly home when medically fit. This will require strategies at a national and regional level to improve access to lower acuity healthcare settings and multi-disciplinary care in the community.

Supplementary Information The online version contains supplementary material available at https://doi.org/10.1007/s00198-021-06294-7.

Acknowledgements We would like to acknowledge the assistance and continued support of the Healthcare Pricing Office, National Office of Clinical Audit, Irish Gerontological Society, Irish Institute of Trauma and Orthopaedic Surgery, clinical leads, local governance committees, local audit co-ordinators, National Irish Hip Fracture Database Governance committee and all patients who have participated in the audit. 
Author contribution HF conceptualised and designed the paper, LB provided the data, JS performed the analysis. All authors drafted the manuscript, provided feedback and approved the final draft.

Funding The Irish Hip Fracture Database is supported by the National Office of Clinical Audit, which is funded by the Quality Improvement Team of the Health Service Executive. No additional funding was received for the preparation of this paper.

Data availability Data from the Irish Hip Fracture Database can be requested from the National Office of Clinical Audit, Ireland.

\section{Declarations}

Ethics approval Ethical approval was received from the Royal College of Surgeons in Ireland Research Ethics Committee (REC202001017).

\section{Conflict of interest None.}

\section{References}

1. Kanis JA, Oden A, McCloskey EV et al (2012) A systematic review of hip fracture incidence and probability of fracture worldwide. Osteoporosis Int 23(9):2239-2256

2. National Office of Clinical Audit (2021) Irish Hip Fracture Database National Report 2020. Dublin: National Office of Clinical Audit. ISSN 2565-5388. Available at https://www.noca.ie/audits/ irish-hip-fracture-database. Accessed 21/10/21

3. Kelly MA, McGowan B, McKenna MJ et al (2018) Emerging trends in hospitalisation for fragility fractures in Ireland. Ir J Med Sci 187(3):601-608

4. Shanahan E, Henderson C, Butler A et al (2016) Dedicated Orthogeriatric service saves the HSE a million euro. Ir Med J 109(4):385

5. Gannon B, O'Shea E, Hudson E (2008) Economic consequences of falls and fractures among older people. Ir Med J 101(6):170-173

6. McGowan B, Casey MC, Silke C et al (2013) Hospitalisations for fracture and associated costs between 2000 and 2009 in Ireland: a trend analysis. Osteoporos Int 24(3):849-857

7. Health Information and Quality Authority (2018) Guidance on a data quality framework for health and social care. In Authority. HIQA (ed) Dublin. Available at www.hiqa.ie. Accessed 21/10/21

8. Walsh M, Ferris H, Coughlan T et al (2021) Trends in hip fracture care in the Republic of Ireland from 2013 to 2018: results from the Irish Hip Fracture Database. Osteoporos Int 32(4):727-736

9. ABF 2020 Admitted Patient Pricelist. DRG Prices for Inpatients and Daycases (2019) Healthcare Pricing Office. Available at www. hpo.ie. Accessed 01/12/21

10. Meagher E, Varghese S, Harty JA, O' Loughlin PF (2021) The decline in incidence rates over a 10 year period: a single centre experience. Injury 52(7):1807-1812
11. Ojeda-Thies C, Sáez-López P, Currie CT et al (2019) Spanish National Hip Fracture Registry (RNFC): analysis of its first annual report and international comparison with other established registries. Osteoporos Int 30(6):1243-1254

12. National Office of Clinical Audit (2020) COVID-19 Report Issue 1.0: Service impact from COVID 19- Analysis based on the Irish Hip Fracture Database June 2019-May 2020. Dublin: National Office of Clinical Audit. Available at www.noca.ie. Accessed $21 / 10 / 21$

13. Golinelli D, Boetto E, Mazzotti A et al (2021) Cost determinants of continuum of care episodes for hip fracture. Health Serv Insights 14:1178632921991122

14. Judge A, Javid MK, Leal J et al (2016) Models of care for the delivery of secondary fracture prevention after hip fracture: a health service cost, clinical outcomes and cost effectiveness study within a region of England. Southampton (UK): NIHR Journals Library

15. Williamson S, Landeiro F, McConnell T et al (2017) Costs of fragility fractures globally: a systematic review and meta-regression analysis. Osteoporosis Int 28(10):2791-2800

16. Ferris H, Brent L, Sorensen J et al (2021) Discharge destination after hip fracture: findings from the Irish hip fracture database. Eur Geriatr Med. https://doi.org/10.1007/s41999-021-00556-7

17. Castelli A, Daidone S, Jacobs R et al (2015) The determinants of costs and length of stay for hip fracture patients. PLoS One 10(7):e0133545

18. Werner M, Macke C, Gogol M et al (2021) Differences in hip fracture care in Europe: a systematic review of recent annual reports of hip fracture registries. Eur J Trauma Emerg Surg. https://doi. org/10.1007/s00068-021-01797-8

19. Kristensen PK, Thillemann TM, Soballe K et al (2016) Can improved quality of care explain the success of Orthogeriatric units? A population based cohort study. Age Ageing 45(1):66-71

20. Ferris H, Brent L, Coughlan T (2020) Early mobilisation reduces the risk of in-hospital mortality following hip fracture. Eur Geriatr Med 11(4):527-533. https://doi.org/10.1007/s41999-020-00317-y

21. Leal J, Gray AM, Prieto-Alhambra D, the REFReSH study group et al (2016) Impact of hip fracture care on hospital care costs: a population based study. Osteoporosis Int. 27(2):549-558

22. Ireland AW, Kelly PJ, Cumming RG (2015) Total hospital stay for hip fracture: measuring variation due to pre-fracture residence, rehabilitation, complications and co-morbidities. BMC Health Ser Res 15:17

23. Salonga-Reyes A, Scott IA (2017) Stranded: cause and effects of discharge delays involving non acute in patients requiring maintenance care in a tertiary referral hospital general medicine service. Aust Health Rev 41(1):54-62. https://doi.org/10.1071/AH15204

Publisher's note Springer Nature remains neutral with regard to jurisdictional claims in published maps and institutional affiliations. 